\title{
Sonographic Monitoring of Psoriatic Plaque
}

MARWIN GUTIERREZ, MD; EMILIO FILIPPUCCI, MD; CHIARA BERTOLAZZI, MD; WALTER GRASSI, MD, Cattedra di Reumatologia, Università Politecnica delle Marche, Ancona, Italy. Address reprint requests to Dr. E. Filippucci, Università Politecnica delle Marche, Department of Rheumatology, Ospedale “A. Murri,” Via dei Colli 52, Jesi, 60035, Italy. E-mail: emilio_filippucci@yahoo.it J Rheumatol 2009;36:850-1; doi:10.3899/jrheum.080739

Psoriasis is a chronic inflammatory skin disease affecting $1 \%$ to $3 \%$ of the world's population ${ }^{1}$. A considerable proportion of patients with psoriasis will develop a form of inflammatory arthritis known as psoriatic arthritis (PsA). More rarely, PsA develops before the psoriatic lesions. The prevalence of PsA in patients with psoriasis varies from $7.6 \%$ to $36 \%$ according to the different populations studied ${ }^{2}$.

The inadequate response to treatment and the toxicity of traditional treatments opened the doors to the development of new therapeutic alternatives such as tumor necrosis factor- $\alpha$ (TNF- $\alpha$ ) antagonists, which are utilized in patients with PsA and psoriasis for reducing the proinflammatory cell infiltration (macrophages, monocytes, keratinocytes, Langerhan's cells, dermal dendritic cells, mast cells, and activated $\mathrm{T}$ cells) at the skin and synovial level $\mathrm{l}^{3,4}$.

Some authors have demonstrated the utility of ultrasound (US) in PsA ${ }^{5,6}$, but to our knowledge no studies have aimed at assessing the potential of this technique in anti-TNF- $\alpha$ therapy monitoring in patients with psoriatic plaque. We describe potential short-term sonographic assessment in 3 patients with psoriasis on biological therapy.

The first case is a 52-year-old woman with PsA for more than 3 years. Because of the resistance to treatment with methotrexate $(15 \mathrm{mg} / \mathrm{wk})$, she started treatment with infliximab (at $5 \mathrm{mg} / \mathrm{kg} /$ infusion, administered at $0,2,6$, and then every 8 wks). Figure 1 shows the US images taken at the beginning of treatment and 2.5 months later. A dramatic improvement is seen in terms of normalization of epidermis thickness, as shown by grayscale US, and disappearance of the dermis perfusion estimated by power Doppler technique.

The second case is a 32-year-old man, with psoriasis for more than 5 years. Due to the resistance to different therapeutic strategies (i.e., salicylic acid-vaseline, retinoids), he started treatment with etanercept $(25 \mathrm{mg}$ subcutaneous twice/wk). After 5 months, improvement was visible at US examination, showing a clear reduction of the epidermal and dermal thickness and disappearance of the power Doppler signal within the dermis layer (Figure 2).

The third case is a 33-year-old man, with a diagnosis of psoriasis, affected for 3 years. He started therapy with adalimumab (40 mg subcutaneous/wk). One month later, the US followup examination revealed a clear improvement of the morphostructural changes in grayscale (normalization of dermal thickness) and the perfusional status using the power Doppler technique (Figure 3).

Our report provides visual evidence that high resolution grayscale US with power Doppler technique allows a detailed morphostructural and perfusional assessment of psoriatic plaque, showing dramatic changes in patients undertaking biological therapy. Further studies of the validity of US are required to define its role for this purpose.

\section{REFERENCES}

1. Myers WA, Gottlieb AB, Mease P. Psoriasis and psoriatic arthritis: clinical features and disease mechanisms. Clin Dermatol 2006;24:438-47.

2. Salvarani C, Lo Scocco G, Macchioni P, et al. Prevalence of psoriatic arthritis in Italian psoriatic patients. J Rheumatol 1995;22:1499-503.

3. Fuchs BS, Hadi S. Use of etanercept in the treatment of psoriasis and psoriatic arthritis. Rev Recent Clin Trials 2006;1:259-63.

4. Mease P. TNF $\alpha$ therapy in psoriatic arthritis and psoriasis. Ann Rheum Dis 2004;63:755-8.

5. Riente L, Delle Sedie A, Filippucci E, et al. Ultrasound imaging for the rheumatologist. IX. Ultrasound imaging in spondyloarthritis. Clin Exp Rheumatol 2007;25:349-53.

6. Kane D. The role of ultrasound in the diagnosis and management of psoriatic arthritis. Curr Rheumatol Rep 2005;7:319-24. 


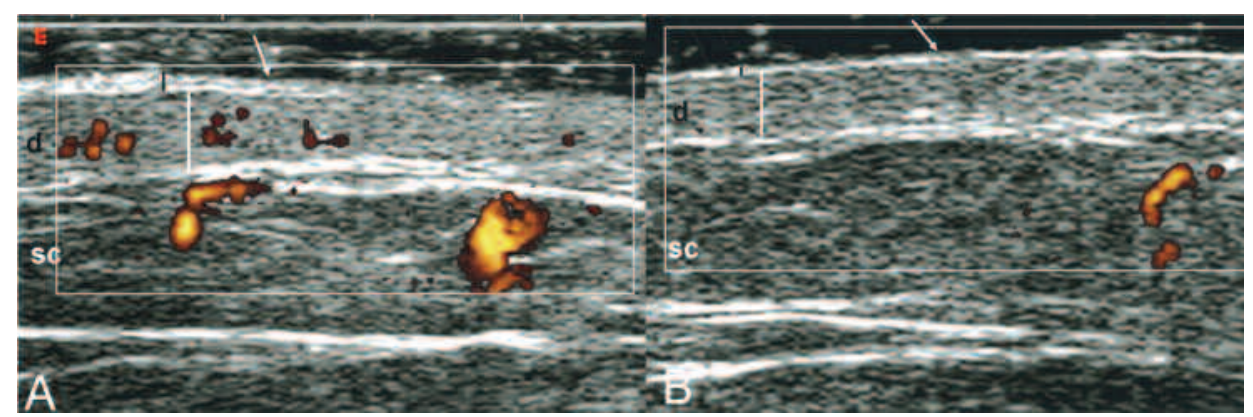

Figure 1. Psoriatic plaque at the abdominal level. Baseline (A) and followup (B) US assessments. A. Grayscale US examination showed marked thickening of both epidermis (arrow) and dermis (d), and abnormal power Doppler signal was found at dermis level. B. 2.5 months after starting treatment with infliximab, US showed reduction of epidermal and dermal thickness (d) and disappearance of the power Doppler signal. Measurements of epidermal and dermal thickness were, respectively, $0.28 \mathrm{~mm}$ and $2.0 \mathrm{~mm}$ at baseline and $0.19 \mathrm{~mm}$ and $1.4 \mathrm{~mm}$ at followup. White and black lines indicate measurements at epidermis and dermis. sc: subcutaneous tissue. US images obtained with a Technos Partner instrument (Esaote Biomedica, Genoa, Italy) equipped with 8-14 $\mathrm{MHz}$ linear transducer. Doppler frequency $12.5 \mathrm{MHz}$.
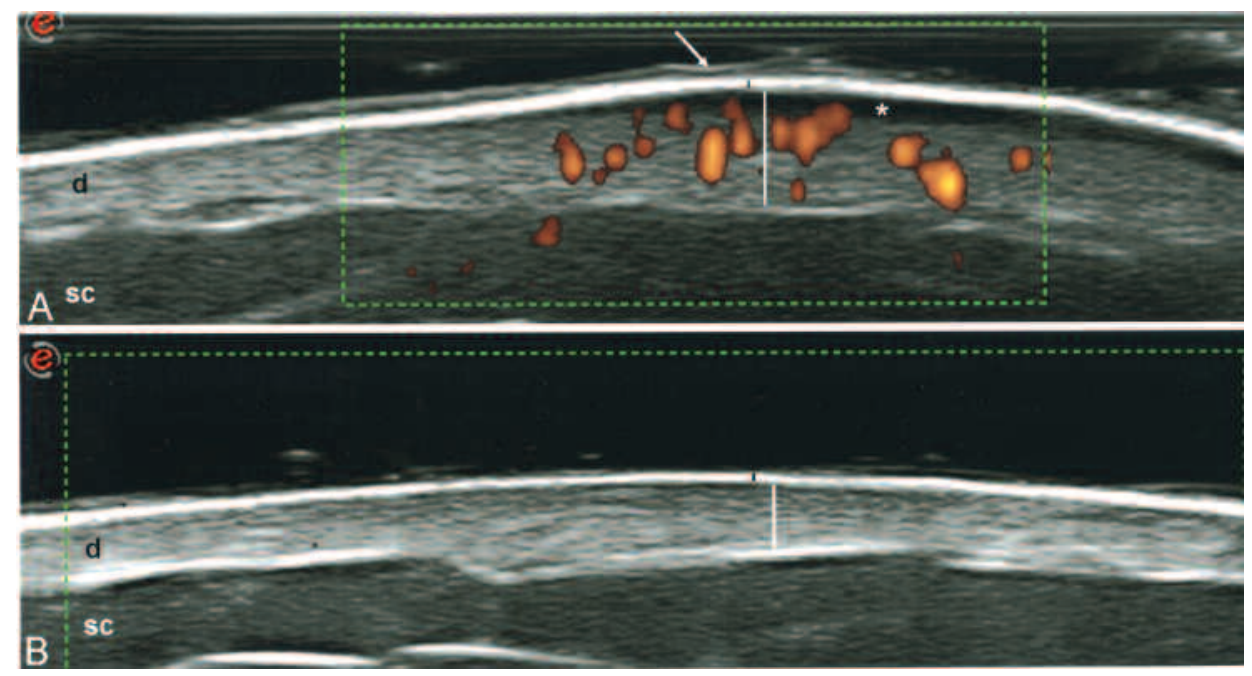

Figure 2. Psoriatic plaque of anterior lower leg. A. Baseline representative US showing marked thickening of both epidermis (arrow) and dermis (d) layer with increased blood flow at dermis level. B. 5 months after therapy with etanercept. A marked reduction of epidermal thickness and complete disappearance of both the anechoic line at the superficial dermis (asterisk) and power Doppler signal within dermis were obtained. Epidermal and dermal thickness measurements were, respectively, $0.32 \mathrm{~mm}$ and $2.1 \mathrm{~mm}$ at baseline and $0.21 \mathrm{~mm}$ and $1.3 \mathrm{~mm}$ at followup. White and black lines indicate where measurements were taken at epidermis and dermis level. sc: subcutaneous tissue. US obtained using a MyLab 70 XVG US system (Esaote Biomedica) with 6-18 MHz linear transducer B-mode frequency of $18 \mathrm{MHz}$ and Doppler frequency 9.1 MHz.

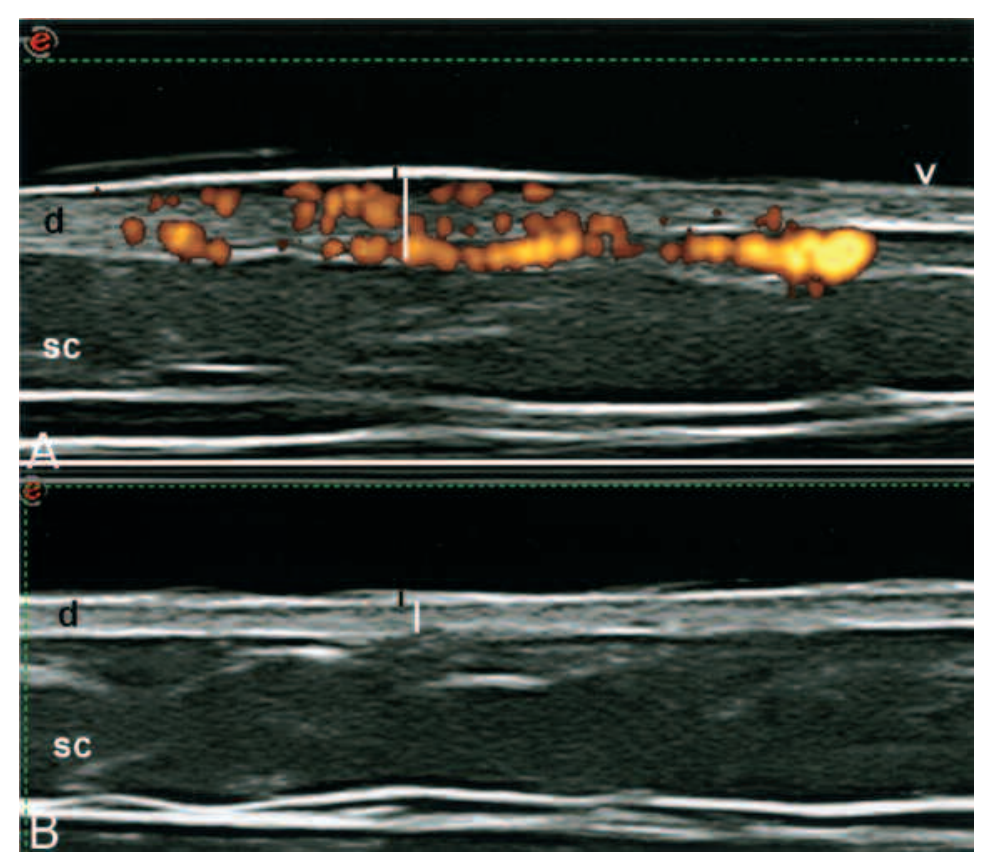

Figure 3. Psoriatic plaque at anterior forearm. A. Baseline US examination shows evident focal thickening of both epidermis and dermis (d) with marked intradermal power Doppler signal. At the normal skin surrounding the plaque, the epidermis appears as a thin hyperechoic band (arrowhead) and dermis shows no power Doppler signal. B. Same patient and same site, 1 month after starting adalimumab. US shows significant reduction of dermis thickness. Disappearance of power Doppler signal within dermis is clearly evident. Epidermal and dermal thickness measurements were, respectively, $0.29 \mathrm{~mm}$ and $1.8 \mathrm{~mm}$ at baseline and $0.23 \mathrm{~mm}$ and $0.56 \mathrm{~mm}$ at followup. White and black lines indicate where measurements were taken at epidermis and dermis level. sc: subcutaneous tissue. US images obtained with a 6-18 MHz linear transducer, MyLab 70 XVG US system, as in Figure 2. 